\title{
Harmonic accents in inference of metrical structure and perception of rhythm patterns
}

\author{
LLOYD A. DAWE, JOHN R. PLATT, and RONALD J. RACINE \\ McMaster University, Hamilton, Ontario, Canada
}

\begin{abstract}
Research on rhythmic structure is somewhat fragmented, due in part to differential use of terminology and a lack of research on the timing of harmonic accents. In this study, a harmonic and a temporal accent were pitted against each other in such a way as to form different rhythm patterns. In addition, two harmonic conditions that varied in the frequency of chord presentations (i.e., the composite rhythm) but not in the frequency of chord changes (i.e., the harmonic rhythm) were presented. Musicians and nonmusicians were requested to report perceived rhythm patterns in an attempt to determine the relative salience of the harmonic and temporal accents. In addition, a behavioral measure of the perceived meter was taken. Results indicated that the location of chord changes was the main determinant of subjects' rhythmic perceptions and the perceived onset of a measure. Furthermore, although subjects primarily inferred different meters based on the composite rhythm, an interaction of metrical and rhythmic choices was found, indicating that perception of rhythm patterns and inference of metrical structure may not always be independent.
\end{abstract}

Although there is a large body of literature on the perception of rhythmic structure in music, certain relevant variables remain uninvestigated. In particular, although the timing of salient harmonic events plays an important part in most musical theories of rhythm, empirical investigations of its role are lacking. This article reports an experiment that was designed as an initial attempt to investigate the timing of chord presentations and changes as cues for the perception of rhythm and the inference of meter.

We have identified two possible reasons for the current inadequate state of research on rhythm. First, the study of rhythmic structure lacks acceptance of a common terminology; and second, there is a lack of studies concerned with the temporal pattern of harmonic accents. The resulting difficulties are discussed in the following sections.

\section{A Definition of Rhythm}

Several researchers employ the term "rhythm" in reference to different phenomena. While it is true that all studies of rhythmic structure can be thought of as analyses of the pattern of time durations that results from various events that occur in a musical flow, researchers often

This research was supported by a predoctoral fellowship from the Natural Sciences and Engineering Research Council of Canada to L.A.D. and by Grant A8269 from the same body to J.R.P. We are indebted to several individuals for suggestions and comments on an earlier draft of this paper; in particular, we would like to thank Kate Kewish, Mari Riess Jones, and two anonymous reviewers for their insightful critiques. Requests for reprints may be addressed to $L$. A. Dawe, Department of Psychology, Social Science Centre, University of Western Ontario, London, ON, Canada N6A 5C2. focus on more specific aspects of music. Generally, thythm refers to any form of reiteration. More specific use of the term has been in reference to the meter-the regular recurrence of grouped strong and weak beats, or heavily and lightly accented tones. Cooper and Meyer (1960) defined rhythm as the way in which one or two unaccented events are grouped in relation to an accented event; and to many, the term is used to refer to the temporal aspects of the duration of individual notes and rests (e.g., Jones, 1987).

Some theorists (Agmon, 1990; Boretz, 1971; Lerdahl \& Jackendoff, 1983) have thought of rhythm as being multi-leveled, with the structure at each rhythmic level being dependent upon the pattern of time spans at lower rhythmic levels. Events that differ in perceptual salience create the hierarchy. Highly salient events serve as defining boundaries for long durations at the upper end of the hierarchy, while less salient events serve as the defining boundaries of shorter time spans lower in the hierarchy. To start with the lowest level, the term rhythm has been used to refer to the surface duration pattern that results from accents associated with the onset and termination of individual notes and rests. We refer to this component of rhythmic structure as the temporal aspect of the musical presentation. In keeping with traditional musictheoretical notions, two intermediate-level duration patterns are of importance for the perception of rhythmic structure (Lee, 1985; Lerdahl \& Jackendoff, 1983; Palmer \& Krumhansl, 1990). First, the regular reiteration of strong and weak beats or pulses and the hierarchical pattern of time spans that results from this regular reiteration are referred to as the "metrical structure." Second, the pattern of durations based on salient timbre-based, temporal, harmonic, and melodic phenomenal events (e.g., chord changes, relatively large interval jumps, and rests) 
that serves as the basis of themes, motives, sections, and phrases is called "phrase structure." Finally, the overall musical structure that results from the interaction of phrase and metrical structure is referred to as "rhythm."

As such, rhythm can be defined as the complex pattern of successive and simultaneous time spans that result from the interaction of a phrase and the metrical structure, both of which are, to varying degrees, themselves dependent on different timbre-based, melodic, harmonic, and temporal events. An adequate delineation of rhythmic structure will depend, therefore, on identification of the perceptually salient events upon which the complex pattern of time spans is built, as well as of the relationship between phrase and meter.

\section{Harmonic Accents: Phenomenal and Structural Accents}

All rhythmic cues can be categorized on the basis of the locus of their perceptual salience. One useful categorization is based on the difference between structural and phenomenal accenting, which was made explicit by Lerdahl and Jackendoff (1983). The distinction is founded upon the view that perception is based on both data-driven (i.e., concerned with phenomenal accents) and conceptually driven (i.e., concerned with structural accents) processing. Phenomenal accents have been defined as any events at the musical surface that give emphasis or stress to a moment in the musical flow (Lerdahl \& Jackendoff, 1983). Examples of phenomenal accents include relatively large interval jumps, contour changes, changes in dynamics, notes of relatively long duration, chord changes, and so forth. Structural accents result from more abstract properties and cognitive principles associated with tonal and diatonic organization. Although not explicitly linked to the accent identifications, the work of Krumhansl and her colleagues offers a general basis from which one might identify structural relationships between various notes, chords, and tonal centers or keys (Bharucha \& Krumhansl, 1983; Cuddy, Cohen, \& Miller, 1979; Krumhansl, 1979, 1990; Krumhansl, Bharucha, \& Kessler, 1982; Krumhansl \& Castellano, 1983; Krumhansl \& Shepard, 1979).

Traditionally, researchers have emphasized the structural facet of a harmonic presentation (e.g., the hierarchical ordering of harmonic stability) rather than the phenomenal aspects of chord presentations. With structural accenting of a chord presentation (e.g., chord progressions), the emphasis is placed on how the harmonic event relates to other chord presentations either in the future or in the past within a tonal context. In contrast, with the phenomenal accenting of a chord change, concern is with the event itself, separate from any tonal relationships. This phenomenal/structural distinction in chord presentations may have some use for investigative purposes. When the phenomenal facet of harmonic presentations is the focus, the timing of chord presentations and chord changes is much more the principal interest than when the focus is strictly on structural relationships.

This study deals with two types of harmonic phenomenal accents: that associated with the presentation of a chord, and that associated with a change of chord. Both types of events are based on a chord's onset, with the distinction resting on whether the chord being presented is the same or different from that previously presented. This distinction, although subtle, is important not only theoretically but also functionally. While it is true that all phenomenal accenting associated with a change of chord is also associated with the accenting resulting from chord onset, the reverse is not necessarily the case. One can have multiple presentations of the same chord. This leads to a consideration of two important constructs in most musictheoretical accounts of rhythm-harmonic rhythm and composite rhythm.

\section{Harmonic Rhythm and Composite Rhythm}

Harmonic rhythm is the sequential pattern of durations provided either by chord changes or by implied triadic patterns within a melody (Piston, 1948; Smith \& Cuddy, 1989). Composite rhythm is the sequential pattern of durations associated with the onset of individual note and chord presentations in a polyphony. To determine the composite rhythm one need only initiate a new timed duration whenever a note in the polyphony begins or ends. In short, the composite rhythm will consist of a series of the notes of shortest duration across the multiple lines of music.

As with the distinction between the two harmonic phenomenal accents discussed above, harmonic rhythm contributes to and is part of the composite rhythm, but the composite rhythm can display serial time patterns not apparent in the harmonic rhythm. Thus, while it is theoretically possible for the harmonic and composite rhythms in a musical presentation to be identical (e.g., when the onset of every note creates a new chord), it is usually the case that the harmonic rhythm represents higher-order temporal relationships.

Several theorists and researchers have focused on the role of harmonic rhythm in the perception of a metrical structure (Cooper \& Meyer, 1960; Piston, 1948; Smith \& Cuddy, 1989). The consensus is that in Western tonal music, harmonic changes coincide with important metrical locations, especially the first beat of the measure. Little empirical research has been conducted on the role of composite rhythm in the perception of meter and phrase. According to several theorists, however, the serial durations created by individual note onsets, in addition to the tempo, will determine the perception of iterative pulses from which one can infer a metrical structure (Lerdahl \& Jackendoff, 1983; Longuet-Higgins \& Lee, 1982, 1984; Longuet-Higgins \& Steedman, 1971; Steedman, 1977). In addition, some theorists (e.g., Lerdahl \& Jackendoff, 1983) believe that the choice of tactus (i.e., the metrical level at which a listener taps his or her foot) and meter is related to the timing of harmonic accents. However, the specific relationship between harmonic and composite rhythm in rhythm perception has yet to be investigated.

In addition to the relationship between harmonic and composite rhythms, two other lines of investigation found in the psychological literature are relevant to this treatise. The first is the notion of accent weights and the role 
of accent coincidence in phrase structure investigations; the second, the focus of metrical-structure investigations, has to do with the nature of meter and its relationship to phrase structure.

\section{Phrase-Structure Investigations}

The literature on rhythmic structure contains several areas of dispute, notably that regarding the role of phenomenal and structural accents in the perception of phrase structure (Jones, 1987; Palmer \& Krumhansl, 1987a, 1987b). Some theorists (e.g., Palmer \& Krumhansl, 1987a, 1987b) acknowledge subtle differences in the perceptual salience of various melodic or temporal events, but insist that the two theoretical component categories combine in an additive fashion to determine the perceived structure. Others (e.g., Jones, 1987) have argued that events either are or are not accents, leaving the notion of accent strength (i.e., perceptual salience) dependent on coincidence of melodic and temporal features, which can then be used to direct attention to anticipated events at regular time intervals (Jones, 1987). Recently, Jones (1992) has modified her views to include the notion of accent weightsan acknowledgment that phenomenal accents can differ in their perceptual salience. She differs principally from Palmer and Krumhansl (1987a, 1987b) in that, according to her updated theory (Jones, 1992), perception of melodic structure is based upon an interactive rather than an additive model of temporal and pitch events.

One of the main difficulties with these theories has been the exclusion of harmonic phenomenal events from both theoretical discussions and investigations of phrase structure. It is possible that harmonic events are perceptually more salient than events within the other theoretical component categories (i.e., timbre-based, temporal, and melodic). Theorists have long emphasized the fundamental role of harmonic functions in traditional Western music. Indeed, it was undoubtedly this emphasis that led to abundant research directed at the structural relationships of harmonic events (e.g., Bharucha \& Krumhansl, 1983; Krumhansl, 1979; Krumhansl et al., 1982; Krumhansl \& Castellano, 1983; Krumhansl \& Shepard, 1979).

\section{Metrical-Structure Investigations}

There is also considerable debate regarding both the basis for metrical structure and its relationship to phrase structure. While most will agree that phrase structure and metrical structure are two theoretically distinct structural components of music, it is questionable whether the two structures are perceptually independent. While some theorists argue that meter is predominantly dependent upon the occurrence of direct phenomenal accents in the microstructure of the music (e.g., Berry, 1976; Cooper \& Meyer, 1960), others claim it is an inferred mental structure (e.g., Benjamin, 1984; Dawe, Platt, \& Racine, in press; Lerdahl \& Jackendoff, 1983; Palmer \& Krumhansl, 1990).

Proponents of the position that metrical structure is perceived directly from the music point to research on the performance of musical compositions which has found musicians to slightly elongate the duration of notes cor- responding to strong metrical locations and to shorten the duration of notes that fall on weak beats (Clarke, 1985; Gabrielsson, 1974, 1987) and to use melodic features such as a subtle change in dynamics to communicate the meter (Schachter, 1980; Sloboda, 1983; Yeston, 1976). There are problems associated with this evidence, however. One could reasonably argue that subtle variations of notes corresponding to metrically significant locations detract from accurate perception of a metrical structure. That is, insofar as a performance fails to produce the precise time ratios upon which a metrical structure is built, perceived meter may differ from the scored meter (Jones, 1990). Arguments that subtle performance cues mark important metrical locations would be strengthened if there was evidence that performers consistently employed them, but the evidence suggests the contrary (Jones, 1990; Sloboda, 1983; Vos \& Handel, 1987). Many of the cues found to occasionally mark metrically important locations are either absent or used to mark phrase boundaries in performance (Palmer \& Krumhansl, 1990), and are thus ambiguous communicators of the meter. Yet more often than not, listeners unambiguously perceive the "correct" metrical structure.

Some theorists believe that meter is independent of phrase structure and is an inferred mental structure (Benjamin, 1984; Lerdahl \& Jackendoff, 1983). Evidence supporting this viewpoint is found in the fact that exact time ratios are rarely, if ever, produced by performers (Gabrielsson, 1986, 1987; Jones, 1990; Palmer, 1989; Palmer \& Krumhansl, 1990). The basis for inference of metrical structure is rarely agreed upon. Lerdahl and Jackendoff (1983) believe that metrical accents arise from the existence of a periodic mental framework or schema. To establish a particular metrical structure, a listener must infer the metrical accents from the location of phenomenal and structural accents in the music. However, according to Lerdahl and Jackendoff, metrical accents themselves are defined as qualitatively distinct types of accents. Similarly, Benjamin (1984) claimed that the role of meter is to give time points an identity independent of the tonal, motivic, or harmonic accents present. Several researchers and theorists (Lerdahl \& Jackendoff, 1983; LonguetHiggins \& Lee, 1982, 1984; Longuet-Higgins \& Steedman, 1971; Steedman, 1977) have suggested various rules that a listener might use to infer meter. Despite differences among these accounts, it is possible to discern a common underlying assumption-namely, that the temporal phenomenal accent of a note of relatively long duration serves as a cue for the perception of metrical structure. In addition, music-theoretical views point to the role of harmonic and composite rhythms, although to date the amount of empirical research that has been conducted to verify the psychological importance of these factors is very small (e.g., Dawe et al., in press; Smith \& Cuddy, 1989).

\section{The Current Investigation}

Many of the issues discussed above were addressed in the present study. With respect to the phrase-structure literature, we were interested in determining the relative 
accent weight of temporal and harmonic accents and the possible role of accent coincidence in defining perceptually salient events in the music. In order to investigate these issues, a harmonic accent (i.e., a chord change) was systematically pitted against a temporal accent. The temporal accent was created by playing a note of relatively long duration $(\mathrm{L})$ followed by four notes of short duration (S). Chord-change locations were systematically varied relative to the location of the temporal accent, resulting in 5 possible harmonically defined rhythm patterns (LSSSS, SLSSS, SSLSS, SSSLS, and SSSSL). Subjects were requested to report the perceived rhythms. Based on subjects' responses, the relative phenomenal accent weight for the temporal and harmonic accents in the phrasing of music could be determined. It was assumed that if the two phenomenal events differed in their perceptual salience, the more prominent event would define the phrase boundary and thus the perceived rhythm pattern. A temporal accent rather than a melodic or timbre-based accent was selected to be pitted against the harmonic accent because of its known role as a boundary cue. With meter, long-duration notes tend to be heard as initiating major metrical units of time (Lerdahl \& Jackendoff, 1983; Longuet-Higgins \& Lee, 1982, 1984; Longuet-Higgins \& Steedman, 1971; Steedman, 1977). In contrast, long durations tend to mark the end of phrases in music (Boltz, 1989; Dowling, 1973; Garner, 1974; Royer \& Garner, 1966, 1970; Vos, 1977; Woodrow, 1909) as well as in speech (Cooper, Paccia, \& LaPointe, 1978; Lehiste, 1975; Oller, 1973; Scott, 1982).

We were also interested in determining the relationship between meter and phrase structure. We wanted to determine (a) whether subjects would consistently and unambiguously infer a metrical structure in the absence of performance cues in the microstructure of the music (as suggested by the work of Essens \& Povel, 1985; Povel, 1981; Povel \& Essens, 1985; and Povel \& Okkerman, 1981 ), and (b) upon what basis such an inference would be made. Specifically, we wanted to determine the relative contribution of composite and harmonic rhythms to the inference of a metrical structure. In order to address this issue, two harmonic conditions that varied in the frequency of chord presentations (i.e., the composite rhythm) but not in the frequency of chord changes (i.e., the harmonic rhythm) were presented. In Figure 1, the composite rhythm patterns are located immediately below the staves. The harmonic rhythm is represented by the grouping below the composite rhythm. While only one example is given in each of the long- and short-duration harmonic conditions, the experiment itself employed five stimuli that differed in the relationship between the harmonic rhythm and the melodic line (see Table 1). The interesting question that arose concerned the meter in which subjects would hear these presentations. With a quarter note followed by 4 eighth notes, a multitude of meters is theoretically possible, but two meters are probable$3 / 4$ and $6 / 8$

If a particular meter was inferred it could also bias subjects to perceive and report rhythm patterns consistent with

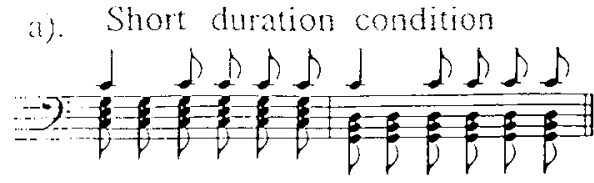

Composite: $\quad D D D D D D D D D D D D$

Harmonic:

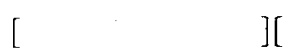

][
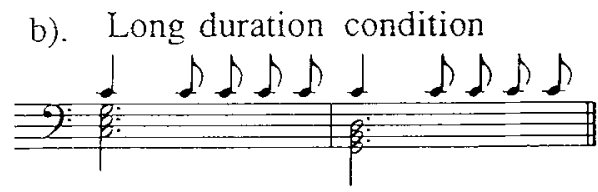

Composite:

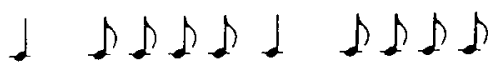

Harmonic:

][ ]

Figure 1. The composite rhythm and harmonic rhythm patterns of stimuli.

that meter. Figure 2 illustrates that the onset of some rhythm patterns, as defined by the location of a chord change, would coincide with accented locations in a $3 / 4$ and 6/8 metrical structure if one assumes that major metrical time spans were inferred to begin with the onset of the temporal accent. This figure also shows an important constraint of metrical structure in traditional Western music, as well as the relationship between $3 / 4$ and $6 / 8$ time. In traditional Western music, meter involves a hierarchy of simultaneous time spans that either double or triple in duration from one lower level to the next higher one. This is evident in the time-span reduction (after Lerdahl \& Jackendoff, 1983) in which dots represent beat events in time.

Movement from the lowest metrical level to the intermediate level can involve the doubling of the duration between beats, which is followed by a tripling as one moves from the intermediate to the next highest metrical level. In this case, one would hear a meter of $3 / 4$ time in which there is a strong accent (indicated by the number of dots beneath each specific location) associated with the onset of the first note (see Example A, Figure 2). Coincidence of the chord change with this metrical accent could bias subjects to report the pattern LSSSS. Two slightly weaker metrical accents would fall on the first and third eighth notes. Chord changes coinciding with these salient metrical locations could bias subjects to report SSSSL (Example B) and SSLSS (Example C), respectively. It should be noted that these accents correspond to each notated beat in $3 / 4$ time (i.e., 3 beats per measure, with a quarter note receiving a beat).

Alternatively, movement from the lowest level to the intermediate level can involve the tripling of the time span 


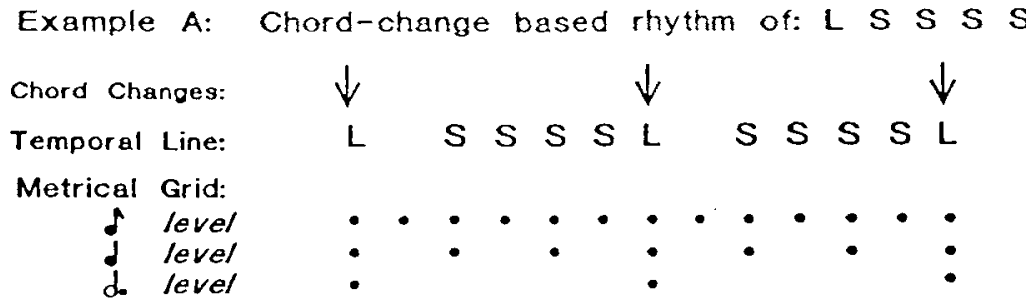

Example B: Chord-change based rhythm of: $S S S S L$ Chord Changes: Temporal Line:

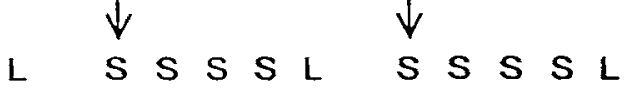

Metrical Grid:

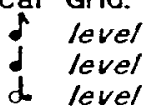

Example C: Chord-change based rhythm of: $S$ S L S S Chord Changes: Temporal Line: L S S S S L S S S S L

Metrical Grid:

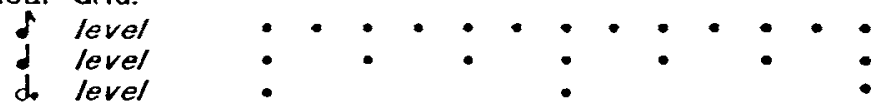

Example D: Chord-change based rhythm of: L $S S S S$ Chord Changes: Temporal Line:

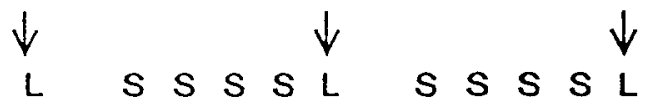

Metrical Grid:

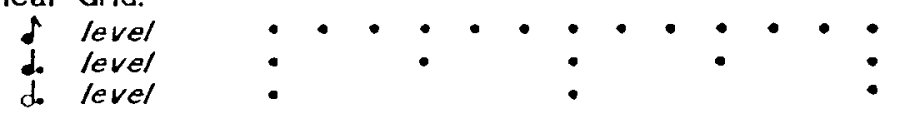

Example E: Chord-change based rhythm of: $S$ S $L S$

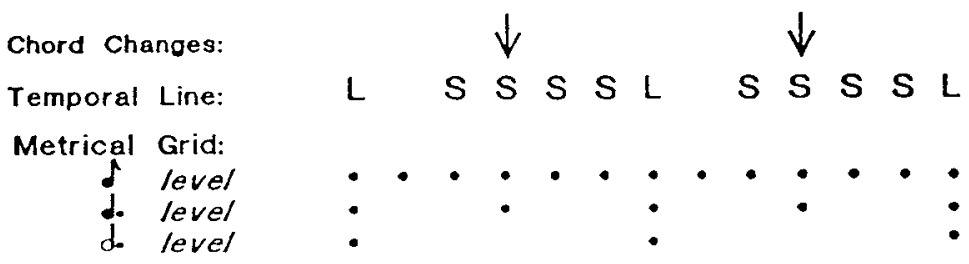

Figure 2. Coincidence of chord-change-based rhythm patterns and metrical accents when major metrical time spans are inferred to begin with the onset of the note of relatively long duration. In Examples A, B, and C, the inferred metrical structure is $3 / 4$ time. In Examples $D$ and $E$, the meter is $6 / 8$ time. 
Example A: Chord-change based rhythm of: L S S S S

Chord Changes:

Temporal Line:

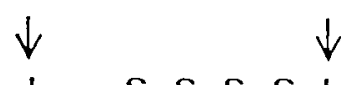

$\downarrow$

Metricel Grid:

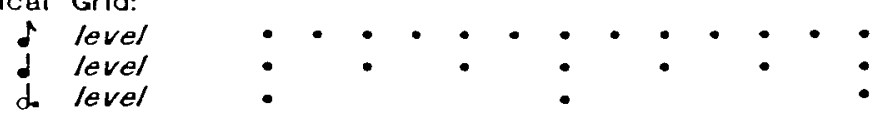

Example B: Chord-change based rhythm of: S S S S L

Chord Changes:

Temporal Line:

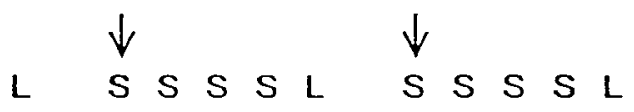

Metrical Grid:

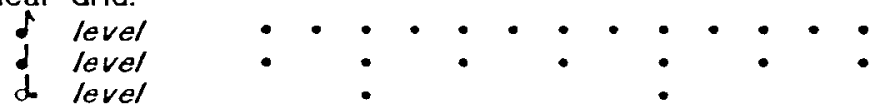

Example C: Chord-change based rhythm of: $S S L S S$

Chord Changes:

$\downarrow$

$\downarrow$

Temporal Line:

L $\quad S$ S S S L S S S S L

Metrical Grid:

d level
d level
d. level

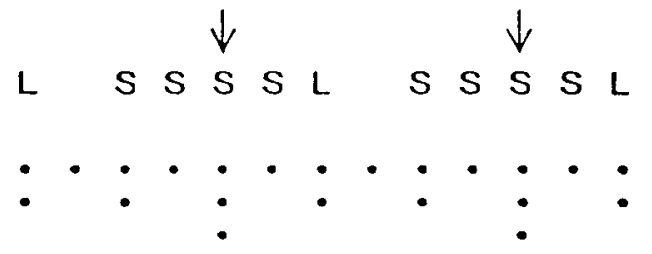

Example D: Chord-change based rhythm of: $L S S S$

Chord Changes:

Temporal Line:

$\downarrow$

Metrical Grid:

$$
\begin{aligned}
& \text { d level } \\
& \text { d. level } \\
& \text { d. level }
\end{aligned}
$$$$
\text { L } \quad S \quad S S S L S S S
$$

Example E: Chord-change based rhythm of: $S S S L S$

Chord Changes:

Temporal Line:

Metrical Grid:

$$
\begin{aligned}
& \text { d level } \\
& \text { d. level } \\
& \text { d. level }
\end{aligned}
$$

$\downarrow$

L $\quad S \quad S \quad S \quad S$ L

$\checkmark$

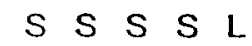

Figure 3. Coincidence of temporal and metrical accents when major metrical time spans are inferred to begin with the location of a chord change. In Examples A, B, and C, the inferred metrical structure is $3 / 4$ time. In Examples $D$ and $E$, the meter is $6 / 8$ time. 
followed by a doubling (see Example D, Figure 2). In this case, one would hear a meter of $6 / 8$ time in which there is a strong accent associated with the onset of the quarter note and a slightly weaker accent falling on the second eighth note. Chord-change-based rhythm patterns of LSSSS (Example D) and SSSLS (Example E) would coincide with these metrical accents. It is important to note that these two metrical accents do not correspond to each notated beat in $6 / 8$ time (i.e., in $6 / 8$ time there are 6 beats per measure, with the eighth note receiving one beat). This is because the tactus of a musical sequence invariably corresponds to an intermediate level of the metrical hierarchy in which the beats pass by at a moderate rate (i.e., only 2 beats per measure; Lerdahl \& Jackendoff, 1983).

As stated earlier, these predictions are based on the assumption that the measure would be perceived to begin with the onset of the temporal accent. However, one of the reasons this investigation was undertaken was to establish whether harmonic and temporal phenomenal accents differ in perceptual salience. Subjects may perceive instead the onset of the measure as coinciding with the chord change. Nevertheless, the inferred meter could still bias subjects to perceive and report rhythm patterns consistent with that meter.

Figure 3 shows that if this were to occur, the same rhythm patterns found to coincide with the locations of metrical accents in Figure 2 would again be found to coincide with metrical accents, but for a different reason. That is, a bias may be found for the rhythm patterns of LSSSS (Example A, Figure 3), SSSSL (Example B), and SSLSS (Example C) with a 3/4 meter because of the coincidence of the temporal and metrical accents. Similarly, a bias for the rhythm patterns LSSSS (Example D) and SSSLS (Example E) may be found with a 6/8 meter.

Finally, we were interested in determining if musical training would result in a difference in the inferred meter and/or a difference in the weighting assigned to the phenomenal accents under investigation. Musical experience and, in particular, formal musical instruction may change one's explicit knowledge and vocabulary of music. Such a knowledge base could alter the way a subject perceives the musical structure through conceptually driven processing of incoming information. Thus, the participants in this experiment were divided into two groups on the basis of whether or not they had ever received formal musical training.

\section{Summary}

This experiment was designed to investigate the roles of two functionally distinct types of harmonic phenomenal accents-those resulting from chord onset and those resulting from chord change-in the perception of rhythmic structure. These accents are associated with the musictheoretical notions of composite and harmonic rhythm, respectively. With its emphasis on the phenomenal aspect of chord presentations, this study departs from traditional approaches to harmonic investigations that have focused on the structural relationship among chord presentations within a tonal context. By systematically varying the location of a chord change relative to a long-duration note and asking subjects to report the perceived structure and meter, several current and relevant theoretical issues were addressed, notably those concerning the relative accent weighting of temporal and harmonic accents and the role of accent coincidence in phrase-structure investigations. The effects of formal musical training on perceived accent weights was also of interest. Finally, the issue of whether or not subjects can unambiguously infer a metrical structure from the composite and harmonic rhythm in the absence of performance cues directly addresses the relationship of meter to phrase structure.

\section{METHOD}

\section{Subjects}

Twenty-four experimentally naive volunteers participated in this study. They were tentatively divided into two groups on the basis of musical training. There were 12 musicians (5 males and $7 \mathrm{fe}-$ males), with a range of 4-11 years and a mean of 6.7 years of formal musical training; their ages ranged from 18-22, with a mean of 20.1 years. Twelve subjects were nonmusicians, with an agerange of 19-21 and a mean of 19 years. Four of these subjects were male and 8 were female. Subjects were recruited from introductory psychology classes, and were given course credit for participating in the experiment.

\section{Apparatus}

The stimuli were generated by a Yamaha CX5M music computer and presented, via a NAD $3020 \mathrm{e}$ integrated amplifier, from two speakers placed approximately 2-3 ft on either side of a video monitor. Each subject sat at a table and responded to the stimuli by moving a joystick that controlled the movements of a cursor visible on a Hitachi 1481 color monitor, situated directly in front of them, at eye-level. The cursor had to be moved into one of five appropriately labeled boxes to indicate the response choice for each trial. Parameters and data for each trial were automatically recorded and stored by the computer on disk. A table and chair were also set up for the experimenter, who sat directly behind the subject.

\section{Stimuli}

The stimuli for this experiment consisted of a temporal presentation and a corresponding harmonic component. On any given trial, one basic rhythm pattern of a long-duration tone ( $L$ ) followed by four short tones (S) was presented 10 times (LSSSSLSSSS . . ). In musical notation, the long-held note was a quarter note (i.e., it received one beat in common time) and the short-duration notes were equal to eighth notes (i.e., they received one half beat in common time). In real time, the long-duration notes were $444 \mathrm{msec}$ and the short-duration notes were $222 \mathrm{msec}$. There were no pauses between notes. All of the 50 tones presented for each trial were Middle $\mathrm{C}$. The reason for presenting all the tones at the same frequency was in order to eliminate melodic phenomenal accents (e.g., contour changes and large interval jumps) and structural accents. Different harmonic presentations accompanied the temporal line. Chords were presented in such a way as to break up the rhythm pattern in relation to the long-duration tone, resulting in one of five possible rhythm patterns (i.e., LSSSS, SLSSS, SSLSS, SSSLS, or SSSSL). The harmonic accompaniment in all conditions consisted of a diatonic cadence (I-V-I) in the key of C Major.

Harmonic accompaniments were presented in a long- and a shortduration condition (see Table 1). In the long-duration harmonic condition, each chord was presented for a duration of 3 beats (i.e., $1,332 \mathrm{msec}$ ) before changing. In the short-duration harmonic con- 
Table 1

A Schematic Representation of Stimuli

\begin{tabular}{|c|c|c|c|c|c|c|}
\hline \multirow[b]{2}{*}{ Condition } & \multirow[b]{2}{*}{ Harmony } & \multicolumn{5}{|c|}{ Temporal Line } \\
\hline & & $\mathbf{L}$ & S S S S L & S S S S L & $S S S S L$ & $\ldots$ \\
\hline \multirow[t]{5}{*}{ Long } & 1 & $\mathrm{C}$ & $G$ & $\mathrm{C}$ & G & $\ldots$ \\
\hline & 2 & C & G & C & G & $\ldots$ \\
\hline & 3 & C & G & $\mathrm{C}$ & G & $\ldots$ \\
\hline & 4 & $\mathrm{C}$ & G & $\mathrm{C}$ & G & $\ldots$ \\
\hline & 5 & $\mathrm{C}$ & $\mathrm{G}$ & $\mathrm{C}$ & $\mathrm{G}$ & $\ldots$ \\
\hline \multirow[t]{5}{*}{ Short } & 1 & $\mathrm{C} \mathrm{C} \mathrm{C}$ & $C \mathrm{C} \mathrm{C} \mathrm{G} \mathrm{G}$ & $G G G G C C$ & C C C C G G & $\cdots$ \\
\hline & 2 & $\mathrm{C} \mathrm{C} \mathrm{C}$ & $C \mathrm{C} \mathrm{G} \mathrm{G} \mathrm{G}$ & $G G G C C$ & $C \subset C G G G$ & $\ldots$ \\
\hline & 3 & $\mathrm{C} \mathrm{C} \mathrm{C}$ & $C \mathrm{G} \mathrm{G} \mathrm{G} \mathrm{G}$ & $\mathrm{G} G \mathrm{C} C \mathrm{CC}$ & $C \subset G G G G$ & $\ldots$ \\
\hline & 4 & $\mathrm{C} \mathrm{C} \mathrm{C}$ & $G G G G G$ & $\mathrm{GCCCCO}$ & $C G G G G G$ & $\ldots$ \\
\hline & 5 & $\mathrm{C} \mathrm{C} \mathrm{G}$ & $G G G G G$ & $\mathrm{CCCCO}$ & G G G G G & $\ldots$ \\
\hline
\end{tabular}

Note-This table is read by following the location of a chord change upward to see where it intersects the temporal line. The five harmonies create five rhythm patterns on the basis of a chord change: LSSSS, SLSSS, SSLSS, SSSLS, and SSSSL.

dition, each chord was presented six times ( $222 \mathrm{msec}$ each) before changing, with each presentation having the same duration as a shortduration note in the temporal line. The only exception to this general description of the harmonic conditions was at the beginning and end of trials, where the duration between trial onset and chord change and between chord change and trial termination was systematically varied to create the five rhythm patterns (see Table 1). It is important to note that the harmonic rhythm patterns are the same in both harmonic conditions, while the composite rhythm patterns differ slightly (see Figure 1). The stimuli were presented through speakers at approximately $72 \mathrm{~dB}$ (SPL), at a tempo of 135 beats per min. This tempo was selected to ensure that any tapping rates (sce Procedure, below) would fall within the preferred range of 200-900 msec (Fraisse, 1963; Handel, 1989), regardless of which metrical level was perceived to be the tactus in either a $3 / 4$ or a $6 / 8$ meter. The timbre was that of an FM-synthesized piano. The temporal line was presented at a slightly greater intensity than the harmonic accompaniment (approximately $4 \mathrm{~dB}$ difference), to facilitate perception of the temporal accent.

\section{Procedure}

There were 60 trials in total, 30 in each of the two harmonic conditions. Each harmonic condition was presented in a blocked format. Half of the subjects received the short-duration harmonic condition first, while the other half received the long-duration condition first. The five different stimuli based on location of the chord change were presented in random order six times. The subjects were instructed to listen to the stimuli and then choose which rhythm pattern they had heard by placing the cursor in one of five appropriately labeled boxes (i.e., LSSSS, SLSSS, SSLSS, SSSLS, or SSSSL). They were under no time constraints, and started each trial by placing the cursor into a startbox. The experimenter ensured that all of the subjects understood what each schemata represented by having them tap each of the five rhythms prior to the presentation of the stimuli. They were further instructed to tap their foot during the presentation of the stimuli. They were also told that there were no correct answers and that it was possible that all trials would sound as if they had identical rhythm patterns. An experimenter sat immediately behind each subject and noted how many times per iteration of the rhythmic pattern the subject tapped his or her foot. Subjects had no difficulty in carrying out this task. Finally, they were required to fill out a standard questionnaire regarding their musical experience and formal training.

\section{RESULTS AND DISCUSSION}

The first result to be noted was that subjects invariably tapped their foot only two or three times per iteration.
It was assumed a priori that if subjects tapped their foot two times per iteration, then they were operating in a $6 / 8$ time meter, and that if they tapped their foot three times, they were operating in a $3 / 4$ time meter. This behavioral consistency was believed to be due to the grouping of iterative pulses established by the harmonic rhythm. While tapping one's foot four or five times per iteration was theoretically possible, such behavior would not be consistent with the pattern set by the composite and harmonic rhythms.

Our first analysis was in order to establish objective support for our arbitrary musical-experience criterion which resulted in subjects being placed into one of two subject groups. Response profiles consisting of percentage counts for the two dependent variables of rhythmic choice (LSSSS, SLSSS, SSLSS, SSSLS, SSSSL) and metrical choice ( $3 / 4$ and $6 / 8$ time) were computed for each subject. These profiles were specified for the five rhythms defined by chord-change locations (LSSSS, SLSSS, SSLSS, SSSLS, SSSSL) in each of the two harmonic conditions (long- and short-duration), resulting in a $10 \times 10$ matrix. The 24 matrices (one for each subject) were then analyzed by means of the hierarchical clustering module in Statistica. The solution, which is shown in Figure 4, supported our original subjective classification. In the figure, our original group of musically experienced subjects, indicated by a solid line, forms a relatively tight cluster on the right side of the solution. Similarly, the nonmusicians, represented by the dotted line, form a cluster on the left side of the solution. The higher level of the connections among the nonmusicians relative to that seen in the musicians' cluster is indicative of greater variability in the pattern of responses for nonmusicians compared with that of musicians. Furthermore, the pattern of responses for two of the nonmusicians was found to be more similar to that found for musicians than that found for their fellow nonmusicians. This is shown in Figure 4, where the two nonmusicians' pattern of responses that were closest to those of the musicians join with the latter's cluster rather than with the nonmusicians' cluster. An investigation of the musical questionnaires completed by these two subjects indicated that they were the only "nonmusicians" with 


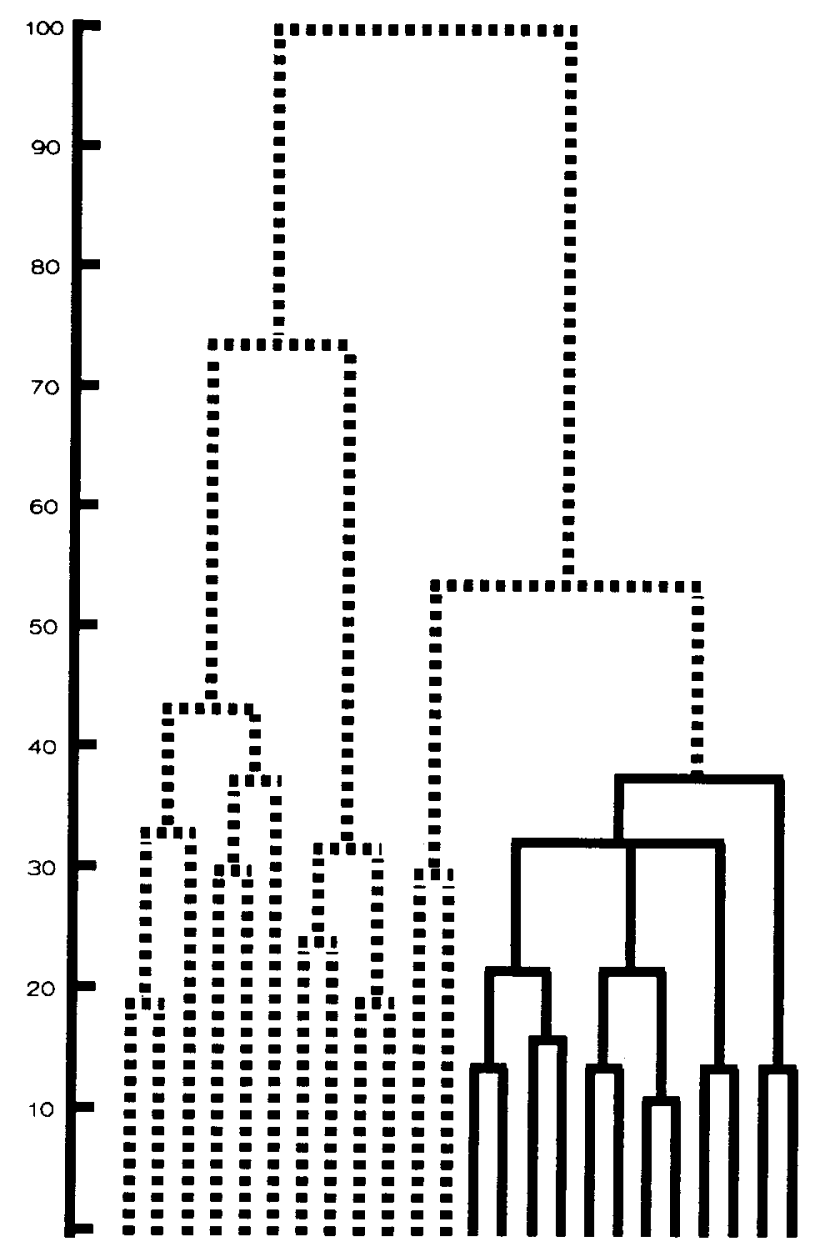

Figure 4. Hierarchical clustering (Ward's method) of the 24 subjects based on the Euclidean distances of individual response profiles. Solid lines represent individuals with formal musical training; broken lines represent nonmusicians. The ordinate represents a proportional measure of dissimilarity relative to the maximum Euclidean distance.

some notable musical experience. They both had some guitar experience, although they did not play often, nor had they had any formal musical training.

Because the measures used in this study were discrete and categorical, a loglinear analysis was performed on the data, with four design and two response variables. The design variables were the musical training of the subject (inexperienced or experienced), the location of the chordchange relative to the long-duration note (resulting in the rhythm patterns of LSSSS, SLSSS, SSLSS, SSSLS, and SSSSL), the duration of the harmonic condition (short or long), and the order of presentation for the two durations. The response variables were the subject's choice of rhythm (LSSSS, SLSSS, SSLSS, SSSLS, SSSSL) and the meter in which he or she was operating ( $3 / 4$ or $6 / 8)$, based on the foot tapping.
The loglinear analysis was implemented with the loglinear module in Statistica. An appropriate model was determined by first conducting tests of partial and marginal association. These tests examine all possible effects and interactions. The test of partial association involved the comparison of a model that incorporated all the equivalent and lower-order interactions, including the interaction under consideration, with a model of all the equivalent and lower-order interactions except the one under consideration. If the difference between the two models was statistically significant, the interaction was retained; if not, it was excluded. The test of marginal association involved the comparison of a model without any interactions with a model that included the interaction under consideration. Again, if the difference between the two models was statistically significant, the interaction was retained. The results of these tests for association indicated that the significant effects were: choice [partial $\chi^{2}(4)=13.19, p<$ .01 ; marginal $\chi^{2}(4)=13.19, p<.01$ ], choice $\times$ training [partial $\chi^{2}(4)=23.82, p<.001$; marginal $\chi^{2}(4)=$ $22.16, p<.001]$, choice $\times$ location [partial $\chi^{2}(16)=$ $462.16, p<.001$; marginal $\chi^{2}(16)=472.71, p<$ .001 ], choice $\times$ duration [partial $\chi^{2}(4)=32.56, p<$ .001 ; marginal $\chi^{2}(4)=13.68, p<.01$, meter $\times$ duration [partial $\chi^{2}(1)=856.81, p<.001 ;$ marginal $\chi^{2}(1)=$ $829.89, p<.001]$, choice $\times$ meter [partial $\chi^{2}(4)=$ 87.80, $p<.001$; marginal $\left.\chi^{2}(4)=77.90, p<.001\right]$, choice $\times$ location $\times$ training [partial $\chi^{2}(16)=106.23$, $p<.001$; marginal $\left.\chi^{2}(16)=107.18, p<.001\right]$, choice $\times$ duration $\times$ training [partial $\chi^{2}(4)=15.10, p<.005$; marginal $\chi^{2}(4)=11.38, p<.05$ ], meter $\times$ duration $\times$ training [partial $\chi^{2}(1)=9.71, p<.005$; marginal $\left.\chi^{2}(1)=15.43, p<.001\right]$, and, finally, choice $\times$ location $\times$ duration [partial $\chi^{2}(16)=40.30, p<.001$; marginal $\left.\chi^{2}(16)=51.26, p<.001\right]$.

The second stage of the analysis first fitted a model that contained only the significant effects from the first stage. The best fitting model was determined by systematically removing each effect and seeing if a significant reduction in the fit occurred. If so, the effect was retained. All possible combinations of effects were systematically fitted by this backward elimination procedure until the best model was obtained. This model contains 4 three-way interactions that account for all of the observed variance: choice $\times$ location $\times$ duration; choice $\times$ location $\times$ training; meter $\times$ duration $\times$ training; and choice $\times$ meter $\times$ location $\left[\chi^{2}(296, N=1,440)=202.11, p=1.00\right]$.

The significant interaction of rhythmic choice with location of a chord change and training, presented in Table 2 , shows that subjects predominantly chose rhythm patterns on the basis of the location of the chord change. With most rhythmic choices along the negative diagonal, the pattern of results for both musicians and nonmusicians indicates several things. First, when the location of the chord change coincided with the onset of the temporal accent (i.e., LSSSS), subjects predominantly chose that rhythm pattern $(51.4 \%$ of the time for nonmusicians and 
Table 2

Effect of Rhythmic Choice by Location of the Chord Change and Musical Training

\begin{tabular}{lrrrrr}
\hline & \multicolumn{3}{c}{ Rhythm on the Basis of the Chord Change Location } \\
$\begin{array}{c}\text { Rhythmic } \\
\text { Choice }\end{array}$ & LSSSS & SLSSS & SSLSS & SSSLS & SSSSL \\
\cline { 1 - 1 } Nonmusicians \\
LSSSS & $51.4 \%$ & $26.4 \%$ & $18.1 \%$ & $16.7 \%$ & $27.8 \%$ \\
SLSSS & $13.9 \%$ & $29.2 \%$ & $13.9 \%$ & $19.4 \%$ & $18.1 \%$ \\
SSLSS & $13.9 \%$ & $9.7 \%$ & $34.7 \%$ & $22.2 \%$ & $16.7 \%$ \\
SSSLS & $11.1 \%$ & $19.4 \%$ & $15.3 \%$ & $29.2 \%$ & $15.3 \%$ \\
SSSSL & $9.7 \%$ & $15.3 \%$ & $18.1 \%$ & $12.5 \%$ & $22.2 \%$ \\
\multicolumn{7}{c}{} \\
LSSSS & $63.9 \%$ & $8.3 \%$ & $11.1 \%$ & $9.7 \%$ & $6.9 \%$ \\
SLSSS & $11.1 \%$ & $50.0 \%$ & $2.8 \%$ & $12.5 \%$ & $15.3 \%$ \\
SSLSS & $8.3 \%$ & $6.9 \%$ & $59.7 \%$ & $9.7 \%$ & $4.2 \%$ \\
SSSLS & $1.4 \%$ & $13.9 \%$ & $15.3 \%$ & $55.6 \%$ & $11.1 \%$ \\
SSSSL & $15.3 \%$ & $20.8 \%$ & $11.1 \%$ & $12.5 \%$ & $62.5 \%$ \\
\hline
\end{tabular}

$63.9 \%$ of the time for musicians), supporting theories of musical structure based on accent coincidence (Benjamin, 1984; Berry, 1976; Jones, 1987). Second, when the rhythm pattern on the basis of the chord change was SLSSS, SSLSS, SSSLS, or SSSSL, subjects chose these rhythms most often $(29.2 \%, 34.7 \%, 29.2 \%$, and $22.2 \%$ of the time, respectively, for nonmusicians, and $50.0 \%, 59.7 \%$, $55.6 \%$, and $62.5 \%$ of the time, respectively, for musicians). In addition, when subjects chose a particular rhythm pattern (e.g., LSSSS), they did so most often when the chord change supported that rhythm pattern. This means that subjects principally used the location of the chord change as a determinant for the perceived rhythm pattern. Finally, musicians and nonmusicians differed in two distinct ways, the first being that, from the greater percentage of responses along the negative diagonal for musicians compared with nonmusicians, it is apparent that the harmonic accent associated with a chord change was perceptually more salient for musicians than for nonmusicians. The other difference is that, compared with musicians, nonmusicians showed a greater bias to select a rhythm pattern on the basis of the temporal accent alone. This becomes apparent when one compares the percentage of responses for the rhythm pattern LSSSS, when the chord-change location supports the rhythm patterns SLSSS, SSLSS, SSSLS, and SSSSL, for musicians and nonmusicians. In all instances, the percentage of responses is higher for nonmusicians than for musicians: $26.4 \%>8.3 \%$ (SLSSS), $18.1 \%>11.1 \%$ (SSLSS), $16.7 \%>9.7 \%$ (SSSLS), and $27.8 \%>6.9 \%$ (SSSSL), respectively. In fact, for nonmusicians, grouping on the basis of the temporal accent (LSSSS) was slightly more salient than was that done on the basis of the rhythm defined by a chordchange location when the chord-supported rhythm was SSSSL $(27.8 \%$ compared with $22.2 \%)$.

The significant interaction of metrical choice by the duration condition of chord presentation and training indicated that subjects were for the most part operating in a $3 / 4$ meter when they were presented with the long-duration harmonic accompaniment and in a 6/8 meter when they were presented with a short-duration harmonic accompaniment. Furthermore, this pattern of metrical responses was more strongly evident in the data of the musicians compared with those of the nonmusicians. When the chord presentation was long, subjects more often than not tapped their feet 3 times per iteration in support of a 3/4 meter $\mathbf{8 7 . 2 \%}$ and $\mathbf{9 2 . 2 \%}$ for nonmusicians and musicians, respectively). In contrast, when the chord presentation was short, subjects were observed to tap their feet more often in support of a $6 / 8$ meter $(81.7 \%$ and $93.3 \%$ for nonmusicians and musicians, respectively).

Because the psychologically meaningful patterns of responses for the two three-way interactions of choice $\times$ location $\times$ duration and choice $\times$ meter $\times$ location are nicely evident in the four-way interaction of choice $x$ meter $\times$ duration $\times$ location, the results can be completely specified by examining the four-way interaction presented in Table 3 . When the duration of the chord presentation was long, subjects predominantly perceived a $3 / 4$ metrical structure (89.7\% of the time). When the perceived metrical structure was 3/4 time under the longduration harmonic condition, the pattern of rhythmic choices on the basis of the location of a chord change was as expected. Subjects chose rhythm patterns predominantly on the basis of a chord change with a bias for the rhythm patterns LSSSS, SSLSS, and SSSSL $(61.0 \%, 55.6 \%$, and $45.7 \%$, respectively). Occasionally (only $10.3 \%$ of the

Table 3

Interaction of Rhythmic and Metrical Choice by the Location of the Chord Change and Harmonic Condition

\begin{tabular}{|c|c|c|c|c|c|}
\hline \multirow{2}{*}{$\begin{array}{l}\text { Rhythmic } \\
\text { Choice }\end{array}$} & \multicolumn{5}{|c|}{ Rhythm on the Basis of the Chord Change Location } \\
\hline & LSSSS & SLSSS & SSLSS & SSSLS & SSSSL \\
\hline \multicolumn{6}{|c|}{ Long-Duration Harmonic Condition } \\
\hline \\
\hline LSSSS & $61.0 \%$ & $13.9 \%$ & $8.3 \%$ & $8.3 \%$ & $13.9 \%$ \\
\hline SLSSS & $4.2 \%$ & $37.5 \%$ & $8.3 \%$ & $13.9 \%$ & $19.5 \%$ \\
\hline SSLSS & $9.7 \%$ & $5.6 \%$ & $55.6 \%$ & $19.5 \%$ & $11.1 \%$ \\
\hline SSSLS & $2.8 \%$ & $6.9 \%$ & $9.7 \%$ & $26.4 \%$ & $5.6 \%$ \\
\hline SSSSL & $13.9 \%$ & $20.8 \%$ & $13.9 \%$ & $12.5 \%$ & $45.7 \%$ \\
\hline \multicolumn{6}{|l|}{ 6/8 Meter } \\
\hline LSSSS & $4.2 \%$ & - & - & - & - \\
\hline SLSSS & $1.4 \%$ & $4.2 \%$ & $1.4 \%$ & $8.3 \%$ & $4.2 \%$ \\
\hline SSSLS & $2.8 \%$ & $11.1 \%$ & $2.8 \%$ & $11.1 \%$ & - \\
\hline \multicolumn{6}{|c|}{ Short-Duration Harmonic Condition } \\
\hline 3/4 Meter & & & & & \\
\hline LSSSS & $8.3 \%$ & $5.6 \%$ & $5.6 \%$ & $2.8 \%$ & $4.2 \%$ \\
\hline SLSSS & - & $5.6 \%$ & $1.4 \%$ & - & - \\
\hline SSLSS & $6.9 \%$ & $2.8 \%$ & $4.2 \%$ & $1.4 \%$ & $2.8 \%$ \\
\hline SSSSL & $1.4 \%$ & $2.8 \%$ & $1.4 \%$ & $2.8 \%$ & $2.8 \%$ \\
\hline \multicolumn{6}{|l|}{ 6/8 Meter } \\
\hline LSSSS & $41.7 \%$ & $15.3 \%$ & $15.3 \%$ & $15.3 \%$ & $16.7 \%$ \\
\hline SLSSS & $19.5 \%$ & $31.8 \%$ & $5.6 \%$ & $9.7 \%$ & $9.7 \%$ \\
\hline SSLSS & $5.6 \%$ & $8.3 \%$ & $34.6 \%$ & $11.1 \%$ & $6.9 \%$ \\
\hline SSSLS & $6.9 \%$ & $15.3 \%$ & $18.0 \%$ & $47.2 \%$ & $20.8 \%$ \\
\hline SSSSL & $9.7 \%$ & $12.5 \%$ & $13.9 \%$ & $9.7 \%$ & $36.1 \%$ \\
\hline
\end{tabular}

Note-Blank spaces and omitted rhythmic choices indicate that no subjects made selections under those specific conditions. 
time), subjects showed evidence of operating in 6/8 time when the chord presentation was of a long duration. Under such conditions, subjects only chose three different rhythm patterns. It is noteworthy that the rhythm patterns SSLSS and SSSSL were never chosen, and that the pattern LSSSS was chosen only when it was supported by a chord change. Subjects predominantly chose the rhythm patterns SLSSS and SSSLS under such conditions. Under these conditions, subjects chose the rhythm pattern SLSSS every time the location of the chord change supported the rhythm pattern SSSSL. This pattern makes sense, given that subjects were operating in a 6/8-time meter. In a 6/8 meter, 3 eighth notes are grouped at the intermediate level of the metrical grid (refer to Figures $2 \& 3$ ). If subjects used the location of the chord change as a metrical cue, then the two rhythm patterns supported by the metrical structure would be SSS-SL and SL-SSS when the chord-changebased rhythm pattern was SSSSL. Subjects chose the latter of these two alternatives. We interpret these findings as evidence that the perceived rhythm pattern biased subjects to infer a meter consistent with that rhythm.

When the duration of the chord presentation was short, subjects predominantly perceived a $6 / 8$ metrical structure ( $87.5 \%$ of the time). When the perceived metrical structure was $6 / 8$ time and the duration of the harmonic condition was short, the pattern of rhythmic choices on the basis of the location of a chord change was as expectedsubjects chose rhythm patterns predominantly on the basis of a chord change with rhythm patterns that coincided with the location of accents in the metrical structure (i.e., LSSSS and SSSLS) receiving the highest proportion of rhythmic response $(41.7 \%$ and $47.2 \%$, respectively). Occasionally (only $12.5 \%$ of the time), subjects showed evidence of operating in 3/4 time when the chord presentation was of a short duration. Under such conditions, subjects only chose four different rhythm patterns. It is noteworthy that the rhythm pattern SSSLS was never chosen under such conditions. Subjects predominantly chose the rhythm patterns LSSSS, SSLSS, and SSSSL under such conditions. Again, we interpret this in terms of a perceived rhythm pattern biasing subjects to select a compatible metrical structure.

These findings are of some importance, particularly for theories of metrical structure. The data clearly support the conclusion that meter is not necessarily communicated through performance cues (Essens \& Povel, 1985; Povel, 1981; Povel \& Essens, 1985; Povel \& Okkerman, 1981). That is, metrically important locations were not marked by phenomenal accents such as a slight elongation of a note's duration or a subtle change in dynamics (Clarke, 1985; Gabrielsson, 1974, 1987). Nevertheless, subjects inferred a metrical structure and then interpreted the musical sequence in terms of that structure.

It is clear that subjects could have used different means to arrive at the inferred meter and perceived rhythm. Specifically, the stimuli contained a number of temporal invariances that subjects could have used. According to Jones's influential work on attentional rhythmicities in music perception, a listener can pick up temporal invariances in the musical flow (i.e., regularly occurring accents) that can then be used to guide attention to future anticipated events. The abstraction of a time interval from unfolding events is a dynamic process based in part on both fulfillment and violation of temporal expectations (Jones, 1990). From this dynamic process emerges a metric of directed attentional resources to which events occurring in the presentation are compared.

In this study such a metric could have been established by the regular occurrence of the temporal accent and/or the harmonic accent of a chord change. The onset of the note of relatively long duration occurred every $1,332 \mathrm{msec}$. If this accent was used to establish an internal rhythmicity of attention, then the harmonic accents could have been judged relative to the regular occurrence of the temporal accent. In other words, it may be the way in which the duration of time established by the regular recurrence of the temporal accent is parsed into smaller time spans by the occurrence of other events (such as chord onsets or chord changes) that is of psychological significance in establishing a meter or rhythm (refer to Figure 2). If the occurrence of a chord change was perceived to coincide with a regular metrical dividing point (Smith \& Cuddy, 1989), the range of probable inferred meters would be reduced.

However, the regular occurrence of a chord change also created a temporal invariance equal to a duration of $1,332 \mathrm{msec}$. In contrast to the previous consideration, therefore, it may be the way in which the time spans created by the chord change are parsed by the occurrence of other events (such as chord onsets and the temporal accent) that is important (refer to Figure 3). If the occurrence of a temporal accent was perceived to coincide with a metrically strong position, the range of probable meters would also be reduced.

Which of these two interpretations is supported by the data? The subjects primarily used the location of the chord change to determine the perceived rhythm pattern. This implies that the harmonic phenomenal accent was more salient than the temporal accent in determining the phrase structure. In terms of the metrical structure, the primary basis for determining an inferred meter was provided by the two harmonic-accompaniment conditions that differed in their composite-rhythm patterns. It was based on the frequency of chord presentations within the temporal or harmonic time span that subjects primarily inferred a $6 / 8$ or a 3/4 metrical structure. In those few instances where selection of the metrical structure was not based on the composite rhythm, it appeared to be based on the rhythm pattern as defined by the location of the chord changes, implying that the chord change was also more salient than the temporal accent for meter determination. Similar conclusions have been made elsewhere (e.g., Dawe et al., in press).

Finally, evidence in favor of the chord change serving as the more prominent accent comes from an observation made during the experimental procedure and from 
reports made by subjects upon completion of the experimental session. In both harmonic conditions, the duration between chord changes was typically $1,332 \mathrm{msec}$. The only exception was at the beginning and end of trials, where the duration between trial onset and chord change was systematically varied to create the five rhythm patterns (refer to Table 1). The result of this manipulation was that some rhythm patterns as defined by the location of a chord change would not be completed at the end of a trial. Interestingly, the experimenter noted that with most such trials, the subjects continued to tap their feet to the projected completion of the rhythm pattern. In addition, subjects reported that some trials appeared to end prematurely (i.e., before they were expected to end). Jones and her colleagues have reported that the way in which a duration is temporally patterned can evoke expectations of when a stimulus should end (Jones \& Boltz, 1989). Thus, we conclude that a chord change was perceptually more salient than a temporal accent in both phrase- and metricalstructure determination.

There are several possible reasons for this finding. In this experiment, only one chord progression was employed (i.e., the I-V-I progression). This was because of our interest in the timing of phenomenal accents, such as chord changes, in the determination of rhythmic structure, excluding structural factors. However, the structural features of a chord progression may affect the perceptual significance of the chord-change location. Structural factors might have been of particular concern in this experiment because of the dissonance created by the suspension of Middle $\mathrm{C}$ in the temporal line while the dominant chord was being played as a harmonic accompaniment. However, early indications from several experiments exploring the contribution of structural accenting to the perceptual salience of chord changes have suggested that structural factors (e.g., significance of a chord progression within a particular key, dissonance, etc.) would not have played a role in the type of rhythm-determination task employed in this study (Dawe, Platt, \& Racine, 1993).

Alternatively, it may simply be that some forms of pitch information (e.g., chord change) are perceptually more salient than temporal information in the determination of rhythmic structure (Dawe et al., in press). Although this may sound somewhat counterintuitive, it has been shown in studies of speech rhythm that when intensity, duration, and pitch contributions to the perceived stress of a syllable are pitted against one another (e.g., Fry, 1958), the fundamental frequency (i.e., pitch) is the dominant stress cue, followed by duration and, finally, intensity features (Handel, 1989). Similar weightings of rhythmic cues may exist in music.

\section{CONCLUSIONS}

Despite the limitations resulting from the use of one chord progression, a number of conclusions can be drawn from this experiment. First, the assumption of equal salience of phenomenal accents in phrase-structure determination was not supported. There was evidence that the location of a harmonic accent was fundamental in establishing a phrase boundary and, thus, the reported rhythm pattern. In addition, musicians showed far less variance than nonmusicians in their tendency to employ the chordchange location as a cue for the rhythm pattern and in their inference of a metrical structure.

Second, support was found for models of phrase structure that rely on the coincidence of phenomenal accents to serve as psychologically important events (e.g., Benjamin, 1984; Berry, 1976; and Jones, 1987). Overall, whenever both the relatively long-held note and the chord change supported the same rhythm pattern, subjects showed a higher percentage of choice for the rhythm pattern based on the accent coincidence (LSSSS) than was evident for rhythm patterns based on either the chord change or the temporal accent alone.

Third, there was evidence for the unambiguous inference of metrical structure supporting conclusions made by others regarding meter determination (Essens \& Povel, 1985; Povel, 1981; Povel \& Essens, 1985; Povel \& Okkerman, 1981). In this study, the meter was determined primarily by the frequency of chord presentation within the time spans defined by the chord changes, supporting conclusions made in another study (Dawe et al., in press) that chord changes serve as important cues for meter determination. Based on the results of this experiment, it seems that music with frequent chord presentations is heard with a faster beat than a piece with the same pattern of harmonic changes (i.e., with the same harmonic rhythm) but fewer chord presentations. This perception of a faster beat can lead to the adoption of a slower tactus and thus the perception of a different metrical structure (e.g., moving from $3 / 4$ to $6 / 8$ time). Once the meter was determined, inferred metrical accents resulted in their location in the music becoming perceptually more salient. That is, we interpret the data in terms of inferred metrical accents serving to heighten attention to events at those locations. In this way, we agree with models such as Jones' $(1987,1992)$, which argue that the occurrence and/ or coincidence of accents may serve to heighten expectations and attention for specific locations.

Finally, perception of rhythm patterns and a metrical structure were not always independent. Subjects had a tendency to select rhythm patterns on the basis of harmonic information (i.e., the location of a chord change) and showed a bias for those patterns consistent with the inferred meter. However, in those cases where the inferred meter was not consistent with the composite rhythm, it was still consistent with rhythm patterns defined by the location of a chord change. Put another way, subjects sometimes inferred a meter on the basis of the rhythm pattern that occurred within the time span defined by a chord change rather than on the basis of the composite 
rhythm defined by that chord change. This suggests that subjects principally used information within the time span defined by the chord change rather than information within the time span defined by the temporal accent to infer the metrical structure.

\section{REFERENCES}

Agmon, E. (1990). Music theory as cognitive science: Some conceptual and methodological issues. Music Perception, 7, 285-308.

Benjamin, W. (1984). A theory of musical meter. Music Perception, $1,355-413$.

BERRY, W. (1976). Structural functions in music. Englewood Cliffs, NJ: Prentice-Hall.

Bharucha, J., Krumhansl, C. L. (1983). The representation of harmonic structure in music: Hierarchies of stability as a function of context. Cognition, 13, 63-102.

BoLTz, M. (1989). Rhythm and "good endings"': Effects of temporal structure on tonality judgments. Perception \& Psychophysics, 46, 9-17.

BoreTZ, B. (1971). In quest of the rhythmic genius. Perspectives of New Music, 9-10, 149-155.

Clarke, E. F. (1985). Structure and expression in rhythmic performance. In P. Howell, I. Cross, \& R. West (Eds.), Musical structure and cognition (pp. 209-236). London: Academic Press.

COOPER, G. W., \& MEYER, L. B. (1960). The rhythmic structure of music. Chicago: University of Chicago Press.

CoOper, W. E., Paccia, J. M., LaPointe, S. G. (1978). Hierarchical coding in speech timing. Cognitive Psychology, 10, 154-177.

Cuddy, L. L., Cohen, A. J., \& Miller, J. (1979). Melody recognition: The experimental application of musical rules. Canadian Journal of Psychology, 33, 148-157.

Dawe, L. A., Platt, J. R., \& Racine, R. J. (1993). Rhythm perception and differences in accent weights for musicians and nonmusicians. Manuscript submitted for publication.

DAwe, L. A., Platt, J. R., \& RACine, R. J. (in press). Inference of metrical structure from perception of iterative pulses within time-spans defined by chord changes. Music Perception.

DowliNG, W. J. (1973). Rhythmic groups and subjective chunks in memory for melodies. Perception \& Psychophysics, 14, 37-40.

Essens, P. J., \& PoVEL, D.-J. (1985). Metrical and nonmetrical representations of temporal patterns. Perception \& Psychophysics, 37, 1-7.

Fraisse, P. (1963). The psychology of time. New York: Harper \& Row.

FRY, D. B. (1958). Experiments in the perception of stress. Language \& Speech, 1, 126-152.

Gabrielsson, A. (1974). Performance of rhythm patterns. Scandinavian Journal of Psychology, 15, 63-72.

GABRIELSSON, A. (1986). Rhythm in music. In J. R. Evans \& M. Clynes (Eds.), Rhythm in psychological, linguistic, and musical processes (pp. 131-167). Springfield, IL: Charles C. Thomas.

Gabrielsson, A. (1987). Once again: The theme from Mozart's Piano Sonata in A Major (K. 331). In A. Gabrielsson (Ed.), Action and perception in thythm and music (pp. 81-104). Stockholm: Royal Swedish Academy of Music Publications.

GARNER, W. (1974). The processing of information and structure. Hillsdale, NJ: Erlbaum.

HANDEL, S. (1989). Listening. Cambridge, MA: MIT Press.

JONES, M. R. (1987). Dynamic pattern structure in music: Recent theory and research. Perception \& Psychophysics, 41, 621-634.

JoNEs, M. R. (1990). Musical events and models of musical time. In R. A. Block (Ed.), Cognitive models of psychological time (pp. 207240). Hillsdale, NJ: Erlbaum.

JoNES, M. R. (1992, February). The structure and function of accent patterns. Paper presented at the 2nd International Conference on Music Perception and Cognition, University of California, Los Angeles.

JoNEs, M. R., BoLTZ, M. (1989). Dynamic attending and responses to time. Psychological Review, 96, 459-491.

KrumhansL, C. L. (1979). The psychological representation of musical pitch in a tonal context. Cognitive Psychology, 11, 346-374.
KrumhansL, C. L. (1990). Tonal hierarchies and rare intervals in music cognition. Music Perception, 7, 309-324.

Krumhansl, C. L., Bharucha, J. J., \& Kessler, E. J. (1982). Perceived harmonic structure of chords in three related musical keys. Journal of Experimental Psychology: Human Perception \& Performance, 8, 24-36.

Krumhansl, C. L., \& Castellano, M. A. (1983). Dynamic processes in music perception. Memory \& Cognition, 11, 325-334.

Krumhansl, C. L., \& ShePARD, R. N. (1979). Quantification of the hierarchy of tonal functions within a diatonic context. Joumal of $E x-$ perimental Psychology: Human Perception \& Performance, 5, 579-594.

LEE, C. S. (1985). The rhythmic interpretation of simple musical sequences: Towards a perceptual model. In P. Howell, I. Cross. \& R. West (Eds.), Musical structure and cognition (pp. 53-69). London: Academic Press.

LEHISTE, I. (1975). The perception of duration within sequences of four intervals. Paper presented at the 8th International Congress of Phonetic Sciences, Leeds, UK.

LERDAHL, F., \& JACKENDOFF, R. (1983). A generative theory of tonal music. Cambridge, MA: MIT Press.

Longuet-Higgins, H. C., \& LeE, C. S. (1982). The perception of musical rhythms. Perception, 11, 115-128.

Longuet-Higgins, H. C., \& LeE, C. S. (1984). The rhythmic interpretation of monophonic music. Music Perception, 10, 424-441.

Longuet-Higgins, H. C., \& SteEdman, M. J. (1971). On interpreting Bach. In B. Meltzer \& D. Michie (Eds.), Machine intelligence (Vol. 6, pp. 221-239). Edinburgh: Edinburgh University Press.

OLLER, D. (1973). The effect of position in utterance on speech segment duration in English. Joumal of the Acoustical Society of America, 54, 1235-1247.

Palmer, C. (1989). Mapping musical thought to musical performance. Journal of Experimental Psychology: Human Perception \& Performance, 15, 331-346.

Palmer, C., \& Krumhanst, C. L. (1987a). Independent temporal and pitch structures in determination of musical phrases. Journal of $E x-$ perimental Psychology: Human Perception \& Performance, 13, 116-126.

Palmer, C., \& Krumhansi, C. L. (1987b). Pitch and temporal contributions to musical phrase perception: Effects of harmony, performance timing, and familiarity. Perception \& Psychophysics, 41, 505-518.

Palmer, C., \& Krumhansl, C. L. (1990). Mental representations for musical meter. Joumal of Experimental Psychology: Human Perception \& Performance, 16, 728-741.

Piston, W. (1948). Harmony (rev. ed.). New York: Norton.

PoveL, D.-J. (1981). Internal representation of simple temporal patterns. Journal of Experimental Psychology: Human Perception \& Performance, 7, 3-18.

Povel, D.-J., \& Essens, P. (1985). Perception of temporal patterns. Music Perception, 2, 411-440.

Povel, D.-J., \& OKKrRman, H. (1981). Accents in equitone sequences. Perception \& Psychophysics, 30, 565-572.

ROYER, F. L., \& GARNER, W. R. (1966). Response uncertainty and perceptual difficulty of auditory temporal patterns. Perception \& Psychophysics, 1, 41-47.

Royer, F. L., \& Garner, W. R. (1970). Perceptual organization of nine-element auditory temporal patterns. Perception \& Psychophysics, 7, 115-120.

SCHACHTER, C. (1980). Rhythm and linear analysis: A preliminary study. In F. Salzer \& C. Schachter (Eds.), The music forum (Vol. 4, pp. 159). New York: Columbia University Press.

ScotT, D. (1982). Duration as a cue to the perception of a phrase boundary. Journal of the Acoustical Society of America, 71, 996-1007.

SLoboda, J. A. (1983). The communication of musical meter in piano performance. Quarterly Joumal of Experimental Psychology, 35, 377-396.

SMITH, K. C., \& CUDDY, L. L. (1989). Effects of metric and harmonic rhythm on the detection of pitch alterations in melodic sequences. Journal of Experimental Psychology: Human Perception \& Performance, 15, 457-471. 
Steedman, M. J. (1977). The perception of musical rhythm and meter. Perception, 6, 555-569.

Vos, P. (1977). Temporal duration factors in the perception of auditory rhythmic patterns. Scientific Aesthetics, 1, 183-199.

Vos, P., \& Hande L, S. (1987). Playing triplets: Facts and preferences. In A. Gabrielsson (Ed.), Action and perception in rhythm and music (pp. 35-47). Stockholm: Royal Swedish Academy of Music Publications.

Woodrow, H. (1909). A quantitative study of rhythm. Archives of Psychology, 14, 1-66.

Yeston, M. (1976). The stratification of musical rhythm. New Haven, CT: Yale University Press.

\section{NOTE}

1. Lerdahl and Jackendoff acknowledge that phrase structure (i.e., grouping) is not strictly hierarchical, although they treat both meter and grouping as such, with grouping overlaps and elisions given special treatment (see Section 3.4, pp. 55-62 of Lerdahl \& Jackendoff, 1983).

(Manuscript received May 6, 1992 revision accepted for publication April 19, 1993.) 